\title{
Dynamic Study of Intelligent Traffic Behaviour Based on Multiple Traffic Modes
}

\author{
Hongwei Jing, ${ }^{1,2}$ Xiaoming Li $\mathbb{D}^{1,2}$ Guangquan $\mathrm{Xu}^{3,4}$ Mengli $\mathrm{Zhu},{ }^{1,2}$ Li Shen $\mathbb{D},{ }^{1,2}$ \\ FangYuan Liu, ${ }^{5}$ and Haoyang Peng ${ }^{6}$ \\ ${ }^{1}$ School of International Business, Zhejiang Yuexiu University, Shaoxing, China \\ ${ }^{2}$ Shaoxing Key Laboratory of Intelligent Monitoring and Prevention of Smart Society, Shaoxing, China \\ ${ }^{3}$ School of Big Data, Qingdao Huanghai University, Qingdao, China \\ ${ }^{4}$ Tianjin Key Laboratory of Advanced Networking (TANK), College of Intelligence and Computing, Tianjin University, \\ Tianjin, China \\ ${ }^{5}$ School of Business, University of Birmingham, Birmingham, UK \\ ${ }^{6}$ School of Business, Marquette University, Milwaukee, WI, USA
}

Correspondence should be addressed to Li Shen; lishen12@zyufl.edu.cn

Received 30 July 2021; Revised 20 August 2021; Accepted 23 August 2021; Published 16 September 2021

Academic Editor: Zhu Xiao

Copyright ( $(2021$ Hongwei Jing et al. This is an open access article distributed under the Creative Commons Attribution License, which permits unrestricted use, distribution, and reproduction in any medium, provided the original work is properly cited.

With the rapid development of society, the traffic problem has become increasingly severe, and the traditional methods can no longer effectively solve the current social traffic behaviour problems. Although studies on the dynamics of human traffic behaviour based on traffic modes can effectively reveal the anomalies in traffic behaviour, few studies integrate intelligent traffic behaviour with multiple traffic modes. Based on the numerous traffic data of bike-sharing and ride-hailing in a Chinese city, this paper reveals the dynamic characteristics of various traffic behaviours in the city by combining spatiotemporal characteristics index and urban spatial structure with human traffic behaviour patterns. The experimental results show that the traffic behaviour of the town presents a double logarithmic power-law distribution in time characteristics, and there is a close interdependent dynamic relationship with the city's spatial structure. The research in this paper can reveal the relationship between bimodal power-law distribution and spatial characteristics in complex systems and help solve social traffic problems effectively in social reality. Further research results can provide practical planning guidance for the behavioural integration of multiple traffic in smart cities.

\section{Introduction}

Since 2005 and 2006, Barabasi [1] and Brockmann et al. [2] have published two papers on human behavioural dynamics in Nature, which initiated a research boom in the emerging interdisciplinary field of human behavioural dynamics. Researchers try to establish a quantitative theory of human behaviour through statistical analysis of many individual or group behaviour data, mining the hidden patterns and rules [3]. In 2009, researchers studied individual and group human behaviour by collecting and analyzing massive human behaviour data. They strengthened or changed people's cognition and understanding of their behaviour and social system activities from a data-driven perspective. Fifteen well-known scholars jointly published an article titled
"Computational Sociology" in Science [4], marking the formation of the interdisciplinary field of data-driven computational sociology.

In the quantitative description of human activities, the traditional work often assumes that human behaviour is randomly distributed over time, approximately conforming to the Poisson process. Because of the importance of the task itself, a queuing theory model based on task priority queue was proposed in [1]. References [5, 6] introduced the time pooling factor into the task queue model. The influence of social factors on the task queue model was considered in [7, 8]. Vazquez [9] proposed a model based on people's historical memory of what they did. A cascaded nonhomogeneous Poisson process model is established in $[9,10]$ to test the periodic rhythms of human behaviour $[10,11]$. 
Considering the influence of interest change factors on human behaviour, Han et al. [12] proposed an adaptive interest-driven model. Quinn et al. [13] analyzed the periodicity of water consumption readings collected from water meters of prominent households every 15 minutes for more than eight months and found that periodicity also existed in people's water use behaviour. Zhou et al. [14] analyzed users' film on-demand records and found that the change of their power index was positively correlated with their activity. The power index ranged from 1.5 to 2.7 , and the change range reached $80 \%$. Hong et al. [15] also found a similar monotonous phenomenon in short message communication interval time. However, Wang et al. [16] found that the power index was not monotonously dependent on the activity but had a slight decline after reaching the peak in the order interval time of online websites. The above studies found that individual activity would lead to significant differences in the characteristics displayed by users or groups of users.

Understanding the spatial characteristics of human behaviour is of great significance to studying complex phenomena driven by human space movement. For example, in the study of traffic flow, the movement of people in different places directly leads to various complex flow phenomena in the traffic network. For instance, Huang et al. [17] analyzed the characteristics of crowd movement behaviour by using the smart cards of 6 million subway passengers and the travel track data of 13,000 taxis. A network mobility method is developed to identify and predict large crowd gatherings, which provides a new way to rationalize urban traffic facilities and prevent and control traffic congestion. The movement of people within and between cities has always been the subject of traffic engineering research. Traffic engineers have long started to collect daily travel data of urban residents utilizing questionnaires and other methods and build models to predict traffic flow based on these data [18]. However, these traditional traffic survey methods often have a high cost, making it difficult to observe and record human space movement behaviour on a large scale and for a long time. With the development of modern electronic technology, more and more means (such as mobile phone positioning systems and GPS) can record the space of individual human motion data for a long time. These data provide statistical methods to study space motion behaviour; based on the research of collective human mobility, Giannotti et al. [19] developed a knowledge discovery method; using the original GPS trajectory data, it is converted into the overall movement mode. Rhee et al. [20] studied the spatial movement behaviours of 44 volunteers carrying GPS terminals in small scenes such as campus, park, urban area, and exhibition hall. The distribution of motion residence time of people moving in a small range presented a power-law through statistical study. Although many scholars have used electronic footprint data to study urban social development and social transportation from space, there are still few studies on solving social transportation problems from human behaviour dynamics. Moreover, most previous studies analyzed and solved problems through the electronic footprint data of a single traffic mode. Few studies used multiple traffic modes to solve traffic problems.

In this paper, the temporal characteristics of traffic behaviour in human behavioural dynamics are emphasized to analyze the material attributes of traffic behaviour of different individuals and groups. In this way, we can find out the regularity of traffic behaviour patterns to further serve intelligent transportation planning. The main contributions of this paper are as follows: First, it analyzes the characteristics of human travel behaviour through the actual data sets of shared bikes and online ride-hailing in a Chinese city. Secondly, based on the analysis of traffic behaviour dynamics under multiple traffic modes, the correlation between traffic behaviour patterns and urban spatial structure is integrated to reveal the characteristics of urban traffic behaviour. Thirdly, based on the random walk model, multiple traffic behaviour patterns are analyzed to reveal the law of group traffic behaviour and serve the intelligent traffic further.

The rest of the paper is organized as follows: Section 2 discusses the appropriate research methods. Section 3 introduces the data set and processing methods. Section 4 presents the commonly used models and optimization algorithms and makes an empirical analysis through actual data sets. Section 5 summarizes the research results.

\section{Related Work}

2.1. Study on Temporal Characteristics of Multiple Traffic Behaviours. With the development of Internet technology, new transportation tools such as online car-hailing and shared bikes have become our daily transportation tools. The movement track of these new vehicles in the urban area has become an essential part of the traffic travel track data. By mining and analyzing the movement track of these vehicles, we can provide a new angle of thinking and a new method to solve the problem of urban social development and social traffic issues.

Many scholars are devoted to studying the parking location of shared bikes. Li and Wang [21] abstract the set of all candidate rental points and their bike lanes into a complex network. Based on the complex network theory, this paper analyzes public bike rental points' location. It uses the complex network theory to analyze which area is the most important. However, the model does not consider the possible network structure and parameters changes after site changes in complex networks. Peng et al. [22] proposed an optimization scheme for key urban public bicycle network nodes by considering the functional properties of public bicycle networks, user travel destinations, and travel distance, combined with critical nodes and wavefront theory. Firstly, the net wave surface flow during peak hours is analyzed to determine the key nodes, and then the nodes are scheduled or added to realize the routine shunting in this area. Finally, the intermediate index is introduced to evaluate the shunting effect. Gao et al. [23] discussed the spatial and temporal characteristics of traditional public bicycle use in Yancheng from the time series clustering of stations. $\mathrm{Zi}$ et al. [24] proposed a new depth graph convolutional network (GCN) model with time attention, predicting the 
bicycle check out/in traffic at each station. The TagCN can simulate the spatiotemporal correlation among different stations and reflect the influence of varying time granularity, namely, the time periodicity at the hour, day, and week levels. With the help of a well-designed time attention mechanism, this model can effectively capture the dynamic time correlation and comprehensive spatial pattern of the bicycle flow.

In recent years, the demand for the online ride-hailing market has increased, and the prediction of rapid growth in demand has become a hot topic. For example, Chen et al. [25] proposed a demand prediction model for online carhailing based on K-means and support vector regression (SVR) and discussed the K-means clustering with the initial longitude and latitude online car-hailing orders as the eigenvalues. The result of clustering is taken as the result of region division. Jia [26] used the ride-hailing data of Nanjing to study the travel behaviour characteristics of residents. Firstly, according to different land types, the core division of Nanjing is divided into 102 independent districts. Then, the analysis of spatiotemporal characteristics of travel demand can dynamically adjust the ever-changing travel demand and traffic supply in a day. Finally, the coupled WAV-SVM model is used to predict the travel demand of residents. The results show that the proposed method has high prediction accuracy and can capture the nonstationary characteristics of short-term traffic demand. In addition, Jiang et al. [27] proposed a short-term demand prediction method for online car-hailing services based on LS-SVM by using least squares and support vector machine (LS-SVM). These are to predict the demand for online ride-hailing to improve passenger satisfaction.

The second part lists relevant research mainly from the perspective of supply and demand. The corresponding prediction model algorithm is used to predict flow, demand, and solve the problem of low traffic resource utilization in the society. People can not meet the traffic demand in a relatively short time, not traffic service, and so on. However, all of these studies directly analyze traffic problems from existing model algorithms and seek optimal solutions. Without human research subjects to diagnose the issues, the root of the issues cannot be found. Therefore, their answers cannot fundamentally eradicate the problems. In the experimental research, from the perspective of the human research centre, we analyze the behaviour pattern of residents in the traffic time of shared bikes and online ridehailing to provide corresponding research support for urban traffic management.

\subsection{Study on Spatial Characteristics of Multiple Traffic} Behaviour. The research on human mobility at the urban scale has always been the focus of many scholars. Mobile phone data and vehicle GPS trajectory data have always been essential means to study human mobility. Barboza et al. [28] used the "call detail record" to identify the Rio de Janeiro metropolitan human mobility patterns. They introduced mobile phone data for transport and spatial modelling of the study area in geographic units to combine aggregated call detail records (CDR) with population data and other sources. The algorithm used to estimate the origin-destination matrix yields a distribution of travel times consistent with the household survey conducted in 2013. Zhao et al. [29] used high-frequency mobile phone location data as the characteristics of first-order Markov processes and quantitatively reproduced individual and population travel patterns on an appropriate time scale based on a unique priority transfer mechanism.

In recent years, the academic interest of researchers has been witnessed in the topic of urban polycentricity. Hu et al. [30] modelled the relationship between traffic interaction and urban function at the road segment level by introducing geographic semantic analysis. First, they built a corpus of road tracks and trained them to obtain semantic embedding representations of road segments. Then, considering the topological connection between sections, the graph convolutional neural network model is used to process the context information and topological information of areas. The social functions along the streets are classified. Based on many accurate taxi trajectory data, a case study was conducted in Beijing, China. The results show that this method has less loss and higher classification accuracy and is superior to other classification methods in road grade. Siangsuebchart et al. [31] used a GPS probe and track gate data for public transportation in Bangkok metropolitan area, Thailand, assessed by human mobility patterns. However, most previous studies have failed to examine the fine-scale regional characteristics of polycenters and how they change with different urban functions at the metropolitan scale. To fill this gap, Liu et al. [32] carefully measured the functional urban multicentre of the Tokyo Metropolitan Area (TMA). They revealed its relationship with the regional characteristics of human mobility patterns through a series of exploratory statistical analyzes. The results demonstrate the static and dynamic design of functional city structures within the original multiperspective analysis framework. References [33-35] explored the universality and diversity of human movement patterns in many cities. In particular, Noulas et al. [34] used the user check-in data of location-based social networking site Foursquare to find that the distribution of human movement step size in 34 big cities was significantly different.

Most of the above studies only use one kind of travel data for analysis. Most of the above studies mainly focus on revealing different behavioural rules through the supply and demand relationship and parking location of online ridehailing vehicles. Few studies show urban traffic behaviours through the integration of multiple traffic modes to help urban planning. In this paper, we take online ride-hailing as the research object, dot the mobile trajectory of online ridehailing in an entire city, analyze the interaction between urban spatial structure and traffic spatial course, and make up for the research gap.

\section{Data Sets}

3.1. Data Set of Shared Bikes. As a bicycle-sharing service provided by enterprises on campus, subway stations, bus stations, residential areas, commercial areas, and public 
service areas, bicycle-sharing is a new environmental protection sharing economy based on time-sharing rental.

The bike-sharing data set adopted in this paper is the actual bike-sharing data set from a northern Chinese city during the week of May 25, 2017, to May 31, 2017. Here, May 25, 26, and 27, 2017, are Thursday, Friday, Saturday, 28, 29, and 30 are rest days (according to the survey, May 30, 2017, is the Dragon Boat Festival; national provisions are 28, 29, and 30, three days off, so Saturday does not rest), and 31, for Wednesday, is a working day. This data set includes working days and holidays simultaneously, so it is of high research significance. We can take this data set as an example to determine the traffic pattern of residents using shared bikes on working days and holidays in this city. The attributes of the shared bikes are shown in Table 1.

The data set has a storage size of 161.69 MB, 1966,727 rows, and six fields (OrderID, UserID, Bike, Bike type, StstartTime, and Geohash-Start). Two types of bike types, bike type $=1$ and bike type $=2$ ( 1 and 2 represent two different shared bikes, resp.). StstartTime records the start time when users use shared bikes. Geohash is an address coding method that encodes two-dimensional spatial longitude and latitude data into a string (in this experiment, we focus on the time characteristics of human traffic behaviour patterns, so this field will not be handled and experimented accordingly).

\section{Experimental Results}

As a new interdisciplinary subject, human behaviour dynamics has not been put forward for a long time, but its important theory and application significance have emerged. At the theoretical level, people have observed many kinds of phenomena of complex systems driven by human behaviour. The study of human dynamics provides new perspectives and new explanations. It is also of considerable application value to mine the statistical characteristics of human behaviour in time and space. Then, taking the traffic aspect of the smart city as an example, the space-time and manager of the statistical traffic attributes used by users can help the society according to their characteristics. Social transportation travel time, frequency of business trips, and scientific and systematic management make transportation very suitable for users' travel habits, improve the utilization of transportation resources, and improve the social happiness of residents. Social managers can see the interaction between users' spatial movement trajectories and urban spatial structure by analyzing its spatial characteristics in this section. For example, in some quarters with large passenger flow, social managers will set up a corresponding number of service areas or gas stations to meet users' needs.

Compared with traditional means of transportation, shared bikes and online ride-hailing have many advantages, which will meet the needs of contemporary society and are an essential part of modern means of transportation. The analysis of shared bikes and online ride-hailing can help us to solve social traffic problems. In the experiment in this section, we conducted a study on the temporal and spatial behaviour patterns of human behaviour through the data
TABLE 1: Data set of shared bikes.

\begin{tabular}{|c|c|c|}
\hline Field names & Field type & Field description \\
\hline Guide & Int & Track ID \\
\hline Companyid & Varchar & The company ID \\
\hline VehicleNo & Varchar & License plate number \\
\hline VehicleRegioncode & Int & Vehicle area number \\
\hline PositionTime & Varchar & Location timestamp \\
\hline UpdateTime & Varchar & Locate the update timestamp \\
\hline Longitude & Float & $\begin{array}{l}\text { Longitude at the time of } \\
\text { positioning }\end{array}$ \\
\hline Latitud & Float & Latitude at the time positioning \\
\hline Speed & Int & Vehicle speed \\
\hline OrderID & Int & Trip ID \\
\hline
\end{tabular}

sets of shared bikes and online ride-hailing and found out different dynamic ways of various traffic. Here, we focus on analyzing time characteristics from the perspectives of periodicity and volatility of users, the time interval of two adjacent traffic behaviours of the same user in various time scales, different group time behaviours under the activity group, paraphernalia, and memory. In space, we dot the mobility data tracks of online hailing vehicles and briefly analyze the interaction between urban spatial structure and traffic spatial movement behaviour. All experiments were conducted on a computer with a Windows 1064-bit operating system, a $3.10 \mathrm{GHz} \mathrm{CPU}$, and $256 \mathrm{~GB}$ of RAM.

4.1. Cycle and Fluctuation Analysis. Cycles and fluctuations in activity are observed in a wide range of human behaviours, and periodicity is sometimes expressed directly in the distribution of time intervals. The movement of an individual is the frequency of a specific behaviour within a unit of time. Intuitively speaking, the daily conduct of human beings should have noticeable fluctuation and periodicity. The study of the periodic and fluctuating behaviour characteristics of human traffic behaviour is helpful to solve the existing traffic problems.

In the experiment of this section, the cycle and fluctuation analysis of people's activity of using shared bikes and online ride-hailing is carried out. Due to the limitations of the data set, the period is not long. The data analysis under the existing data set shows that shared bikes take one week as a unit, which may be cyclical. Shared bikes and ride-hailing are active and frequently used during the commuting hours of the day. Figures 1 and 2, respectively, show the statistics of the number of times users used shared bikes for 24 hours from May 25, 2017, to May 31, 2017, and the number of times users used online car-hailing within 24 hours from December 20, 2019.

In Figure 3, the abscissa is the date, and the ordinate is the total number of shared bikes used per hour. As shown in Figure 3, when people use shared bikes to travel, the average number of times on weekdays is more significant than on holidays. In rush hours, people use shared bikes more often on weekdays than on holidays. In addition, the number of people using shared bikes shows a periodic fluctuation distribution in a cycle of working days and holidays. On the first day after the holiday, the number of people using shared 


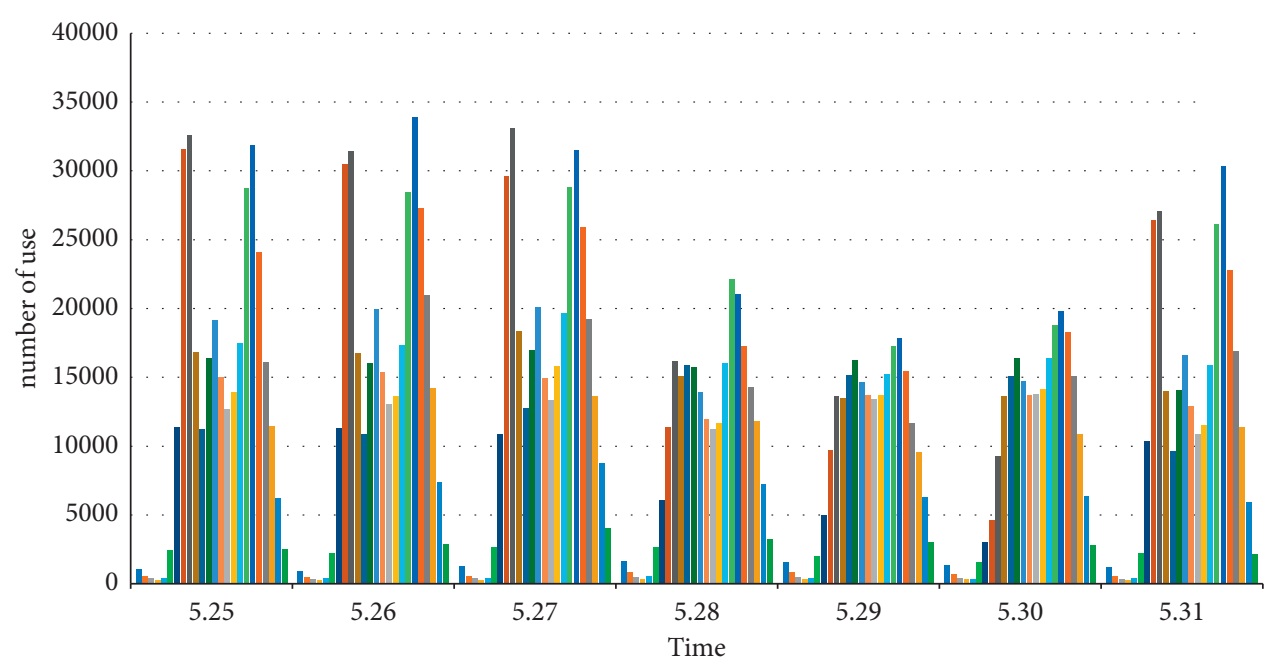

Figure 1: The time interval distribution of the same user using a shared bike for two adjacent times.



FIgURe 2: The time interval distribution of the same user using a shared bike for two adjacent times.

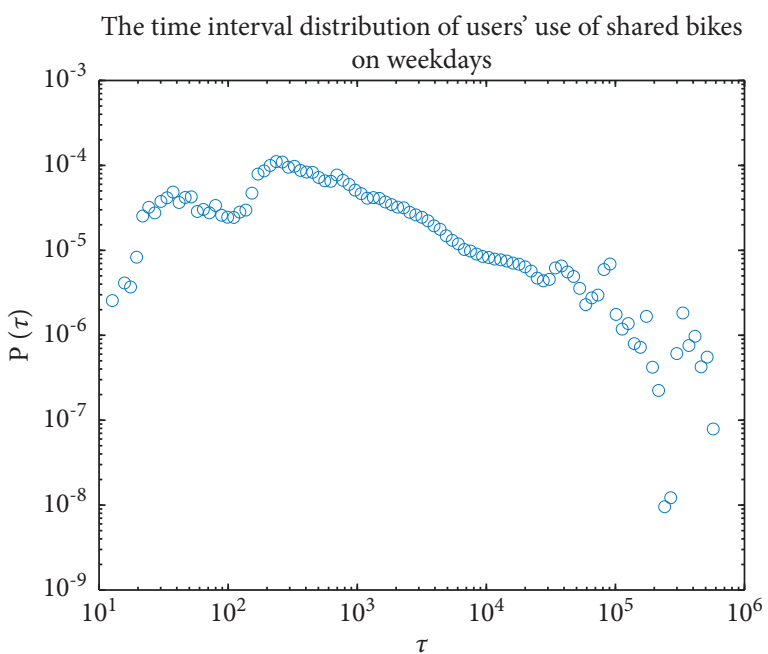

(a)



(b)

FIGURE 3: Number of times of using shared bikes (5.25-5.31). 
bikes is lower than that of ordinary working days, and the gap is the largest in rush hours.

The abscissa is time, 24 hours a day, and the ordinate is the total number of times using e-hailing vehicles per hour. As shown in Figure 4, if one day is taken as a cycle, there is no periodicity in the number of times people use online carhailing. The number of times people use online ride-hailing is meagre from 1 to $7 \mathrm{am}$, but it increases sharply from 7 to 9 am and drops sharply from 9 to $11 \mathrm{am}$. From 12 to 22, there was a stable fluctuation, in which the usage times of two hours (19 and 20) were the most during this period and the most during the whole day. Then people's usage times peaked at 19 o'clock and began to decline.

4.2. Multiple Traffic Time Interval Analysis. In studying the time characteristics of human behaviour dynamics, the time interval distribution forms of human behaviour in different systems are different. In this section, the time characteristics of multiple vehicles are analyzed mainly through

$$
P(\tau) \propto \tau^{-\alpha},
$$

where $P$ is the probability, $\tau$ is the interval time between two adjacent behaviours of people, and $\alpha$ is its power.

To eliminate the fluctuation and disarray phenomenon in the tail of the empirical result $P(\tau)$ in the logarithmic coordinate system, we use the static Binning method [36] in the statistical analysis to observe the attenuation trend and calculate the power exponent $\alpha$ in the logarithmic coordinate system.

4.2.1. Sharing a Bike. Here, we mainly consider holidays and working days to distinguish between periods and people's demand for shared bikes. There are also two types of shared bikes in the data set, namely, type $=1$ and type $=2$. Therefore, cross-analysis is conducted according to different categories. Figure 1 shows the time interval distribution diagram of the same user using the shared bike two times adjacent when type $=1$ on workdays and holidays.

The abscissor of Figure 1 is $\tau$, which stands for the time interval between two adjacent rides of the same user in seconds. $P(\tau)$ is the probability of people using shared bikes at different time intervals. Whether on weekdays or holidays, the time interval of the same user using the shared bike two times adjacent presents a bimodal power-law distribution in the log-log coordinate system. What is remarkable here is that no matter how we change the time characteristics in the experiment, the shared bike will show an apparent double peak phenomenon. Although the distribution curve of the two is roughly the same, compared with working days, we can see that the point to the left of the first peak of the function curve of holidays is more continuous than that of working days. The extreme point of the first peak is close to 10-4 square, and there is a slight warp at the end of the function curve of working days. But the end of the workday is more disorganized than the end of the holiday.

Before in the work of this paper, we read a lot of related research studies on the dynamics of human behaviour and found that human behaviour time interval presents a power- law distribution or with heavy tail phenomenon shows drab exponential distribution, and we did not see any research that is similar to ours; this piece of research results has two obvious peaks of a power-law distribution. Therefore, we focus on this research. The study found that many major cities in China currently have one broken bike, and it is likely that there are several broken bikes at a parking spot. So, when a user opens a bike and realizes it is broken, they immediately switch to another one, so this is the first peak around $\tau=35$, which is about the time a user would find a car broken and change to another one. This survey explains the first peak. In our investigation, we discovered that there is more than one reason for the second peak. One of the most crucial first reasons is that with the mass production of shared bikes in the last decade and the incorrect use of the user and parking personnel's rude behaviour, there is widespread damage of shared bikes in some areas. As a result, a city resident who wants to use a shared bike to get around cannot even find one that is not damaged and works properly at a shared bike stop. The second reason is that, with the continuous improvement of public transport facilities, long-distance roads can be solved by buses or subways, while shared bikes mainly solve the "last kilometre problem." For example, when urban residents are on their way to work, they find that there are many cars and they are crowded in some sections near their work point, so they will use shared bikes to travel. However, some areas may have not only a large traffic flow but also a large flow of people. At this time, people will choose to walk. Again, people will look around for bike-sharing parking places and use the bikes to get around. $\tau$ corresponding to the second peak is about 250 seconds, which can well explain this social phenomenon.

Figure 1(b) is the time interval distribution of the same user who uses the shared bike two times adjacent to each other on weekdays and holidays for the shared bike model type $=2$.

In Figure 2, we can see the same phenomenon as in Figure 1, which indicates that no matter what type of vehicle is, there is a common feature between them, which is consistent with the above.

Because the Dragon Boat Festival is a traditional Chinese festival, this festival has a special meaning for Chinese people. Therefore, under the condition of holidays, it is further subdivided into holidays during the day (8:00-17:00), holidays before midnight (18:00-23:30), and holidays after midnight (0:00-6:00). To better verify the activity characteristics of the shared unit at different times, the experiment did not classify the types of shared bikes. Still, it used two types of shared bikes for comparative analysis. Figure 5 shows the time interval distribution diagram of the same user using the shared bike two times in the daytime, before midnight, and after midnight of the holiday, respectively.

It can be seen from the figure that the points at the end of the power-law distribution are more scattered on holiday nights than in the daytime. One can also see that people's activities and behaviours on holiday nights are more diversified and random. Meanwhile, there is almost no function curve on the left side of the first peak in Figure 5(c), while the function curve on the left side of the first peak in 
The time interval distribution of users' use of shared

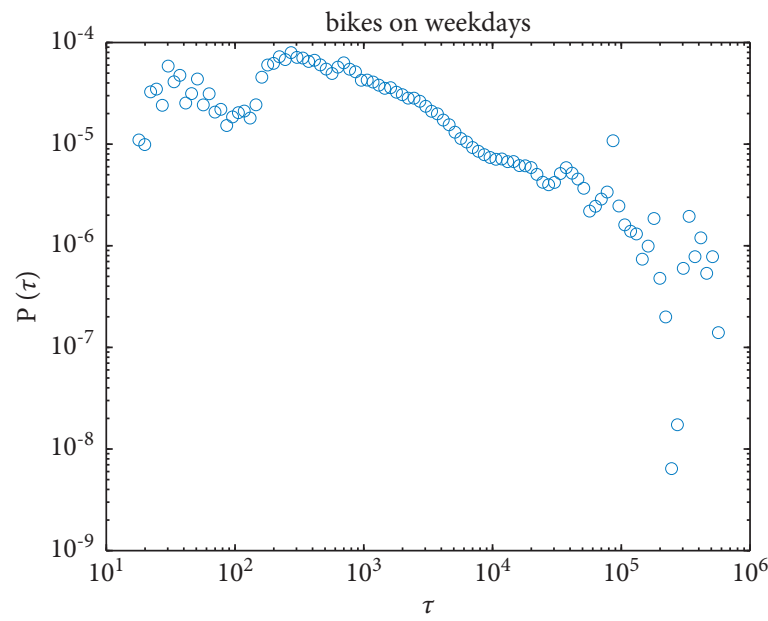

(a)
The time interval distribution of users' use of shared bikes during holidays

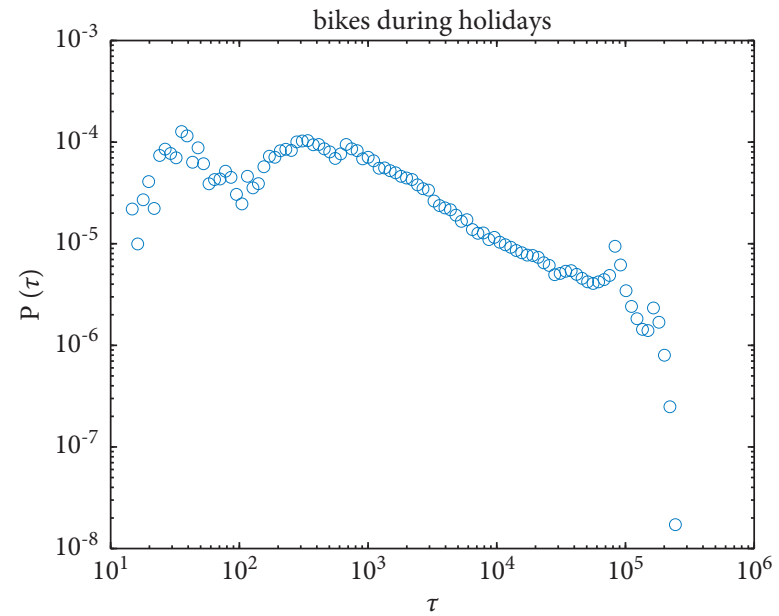

(b)

FIGURE 4: Statistics of times of users using online car-hailing (in hours).

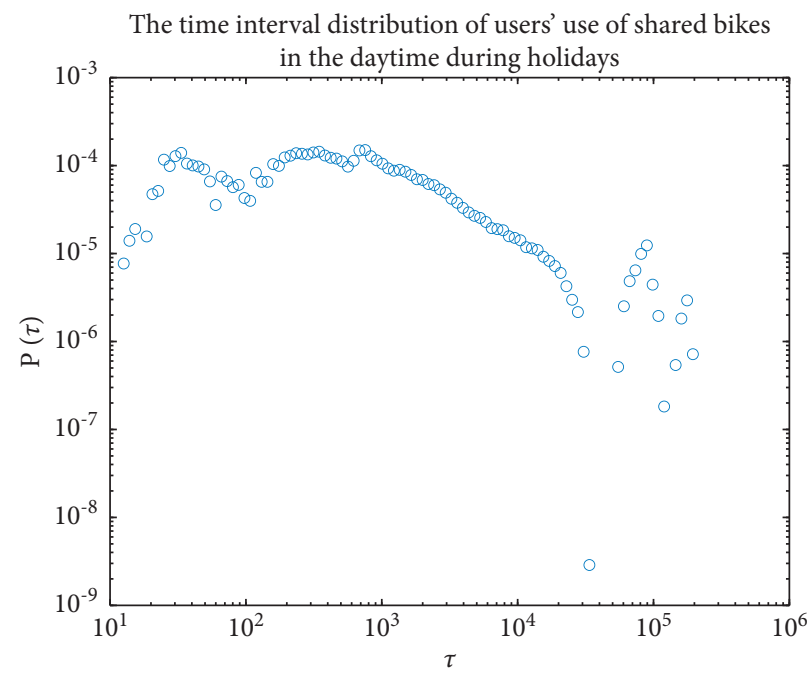

(a)

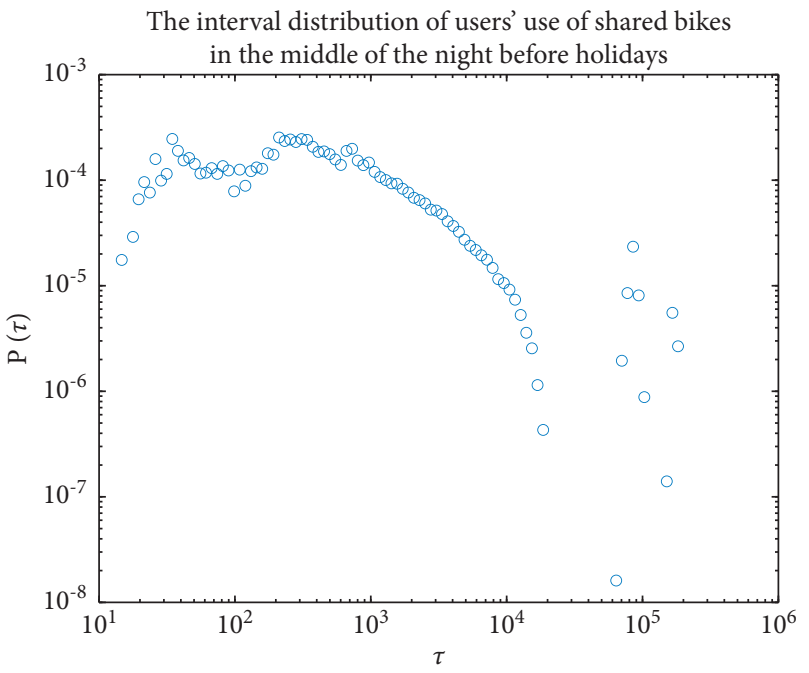

(b)

The time interval distribution of users using shared bikes

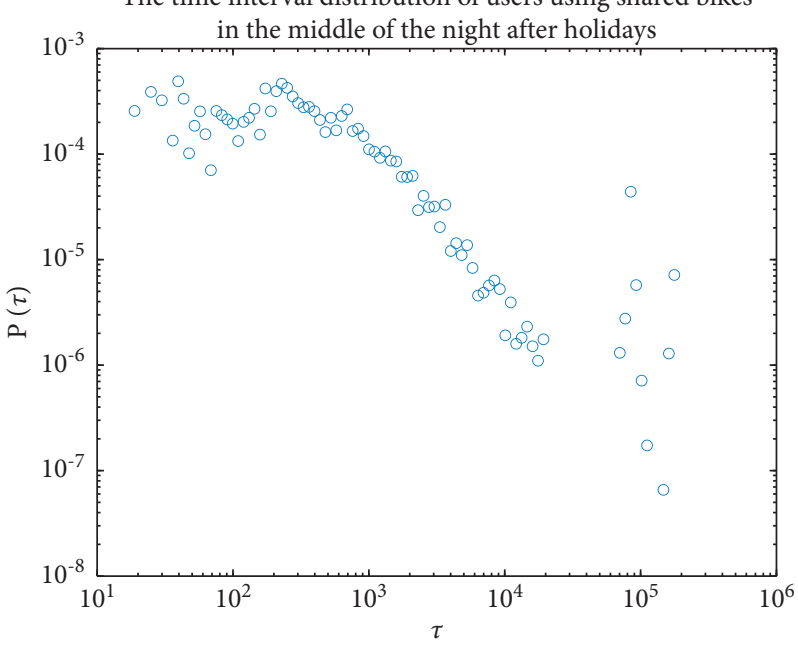

(c)

Figure 5: Activity characteristics of the shared unit during the festival. 
Figures 5(a) and 5(b) is complete compared to that in Figure 5(c).

In the above empirical analysis of the periodic distribution of shared bikes, it was found that, in a certain period in the morning and a certain period in the afternoon of workdays, the number of users using shared bikes also reached two peaks in a day. Therefore, further investigation of these two periods in the city shows that these two periods correspond to the rush hour. According to the survey, the city's peak hours for going to work are from 7:00 am to 9:00 am and 4:00 pm to 7:00 pm (Figures 6(a) and 6(b)), respectively, corresponding to the time interval distribution of the same user using the shared bike two times adjacent to each other during the rush hours and the rush hours after work.

Figure 6(a) finds the first peak remarkably incomplete, while Figure 6(b) is quite complete. There is a relatively straightforward inflexion point at the end of the function curve in Figure 6(a) at $\tau=1400$ and $P(\tau)=3.17 e-5$, while there is no such distribution phenomenon in Figure 6(b).

4.2.2. Network about Cars. In this part, we use the ridehailing track data set of a northern city in China to make a comparative analysis of the power-law curves of the time interval between the two adjacent trips of ride-hailing users and the time interval between users using different ridehailing companies. In Figures 7 and 8 , the $x$-coordinate is $\tau$ (time interval), and the $y$-coordinate is $\mathrm{P}(\tau)$ (the probability of the event occurring at the corresponding time interval).

From the test in Section 4.1, we find that the number of times people in this city use online car-hailing is very few from 1 to $7 \mathrm{am}$, increases sharply from 7 to $9 \mathrm{am}$, and drops sharply from 9 to $11 \mathrm{am}$. From 12 to 22 , there was a relatively stable fluctuation, in which the usage times of two hours (19 and 20) were the most during this period and in the whole day. Then people's usage times reached the peak at 19 o'clock and began to decline. To further reveal this phenomenon and better serve traffic decision-making, this section selects two representative periods to carry out an experimental analysis of power-law distribution. The details are shown in Figure 7.

Figures 7(a) and 7(b), respectively, show the same user's time interval distribution diagram using the e-hailing car for two consecutive times in the two break periods of 7:00 to $11: 00$ and $12: 00$ to $24: 00$ on this day. The time interval distribution is discontinuous from 7 to 11 and from 12 to 24 . There is a break at $260 \leq \tau \leq 346,826 \leq \tau \leq 1003$, forming a three-segment function curve. The whole time interval distribution presents a two-segment power-law distribution.

The ride-hailing data set contains different company IDs. Figure 8 shows whether there is a difference in the time interval distribution between the two adjacent times of the ride-hailing services of other companies.

In Figure 8, we fit the time interval distribution of the four companies, and we can see that the power exponents of different companies are different. The power index is about 2.17 , company $\mathrm{B}$ is about 1.08 , company $\mathrm{C}$ is about 2.96 , and company $\mathrm{D}$ is about 1.95 . Other power indexes indicate the user's activity to perform (activity represents the frequency of the user group's behaviour). The higher the power index, the higher the activity. Therefore, through the analysis of the power index of these four companies, we find that users use company $\mathrm{C}$ for online ride-hailing with the highest frequency and company $\mathrm{B}$ with the lowest frequency. This phenomenon can support the government to carry out accurate and effective regulation and control of the online ride-hailing market. For example, the government can learn people's behavioural preferences by comparing the power index of different companies. According to this behavioural preference, it can, on the one hand, increase efforts to promote the development of the e-hailing companies favoured by users and provide corresponding help and support to the e-hailing companies not favoured by users. On the other hand, the government can also redistribute the vehicle transportation resources of these companies to make them more efficient.

The time interval distribution of different companies shows other characteristics. This section analyzes the time characteristics of bike-sharing and ride-hailing when used and finds that they are more inclined to obey power-law distribution in the double logarithmic coordinate system, which is of guiding significance for the placement of multiple vehicles in cities. For example, through the analysis of the comparative study of different companies, we can according to users use different network about car company network about car service interval and network planning about the timing of the car driver of the car and the time interval, so that it can reduce the waiting time for users; users of the happiness index of ascension also can reduce the network about car driver unnecessary waiting time. It could also give ride-hailing drivers more time to rest.

It should be mentioned here that Figure 7 studies the data sets of all online ride-hailing services due to different research conditions. In contrast, Figure 8 shows the data sets of online ride-hailing services of different companies. It can be seen from Figure 8 that there are significant differences in the power-law function curves of other companies, as well as the differences in power indexes. Figure 7 finds the time characteristics of urban residents' use of e-hailing, while Figure 8 compares the difference in time characteristics of users' use of e-hailing from different e-hailing companies. Therefore, these two experiments can be regarded as separate experiments with little correlation with each other.

4.3. Online Ride-Hailing Data Set. For ordinary users, online ride-hailing has many advantages, such as safety, short waiting time, arbitrary taxi places, and transparent fees. The ride-hailing data set adopted in this paper datum set in the same city as the shared bike. The data set adopts the accurate online ride-hailing track data set of 24 hours on December 20,2019 . The original data set is the online ride-hailing track data of each area of the city recorded every 5 minutes. There are $288 \mathrm{CSV}$ files in total, with an overall size of $1.97 \mathrm{G}$. This trajectory data set specifically involves the trajectory of each online hailing car equipped with GPS devices in the city, mainly including company ID, license plate number, vehicle 


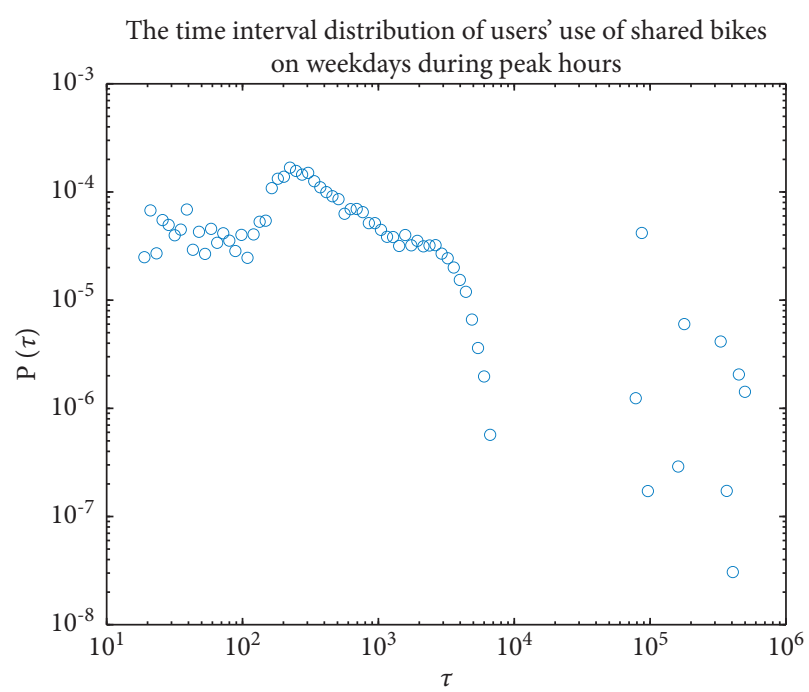

(a)

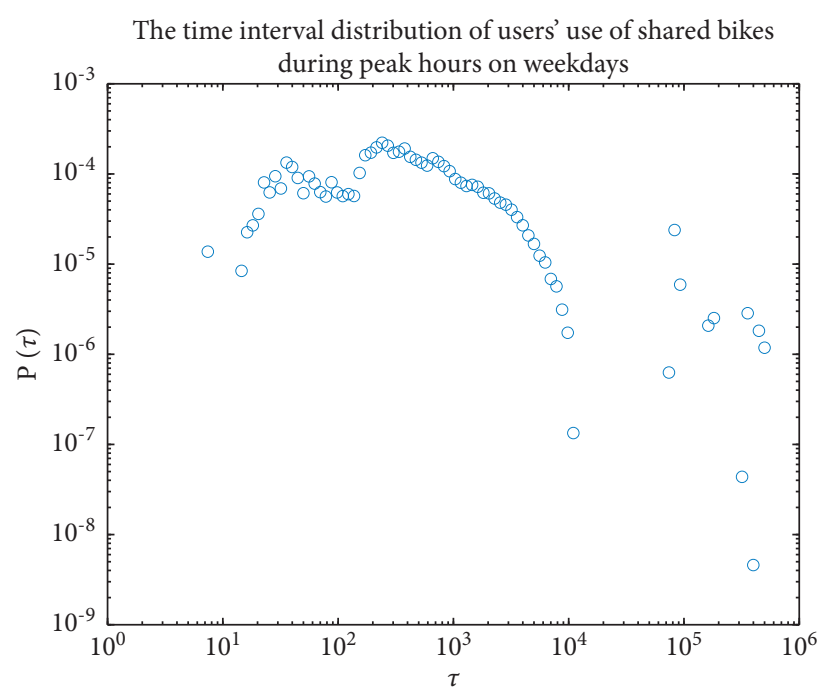

(b)

FIGURE 6: Shared unit time characteristics during rush hour.

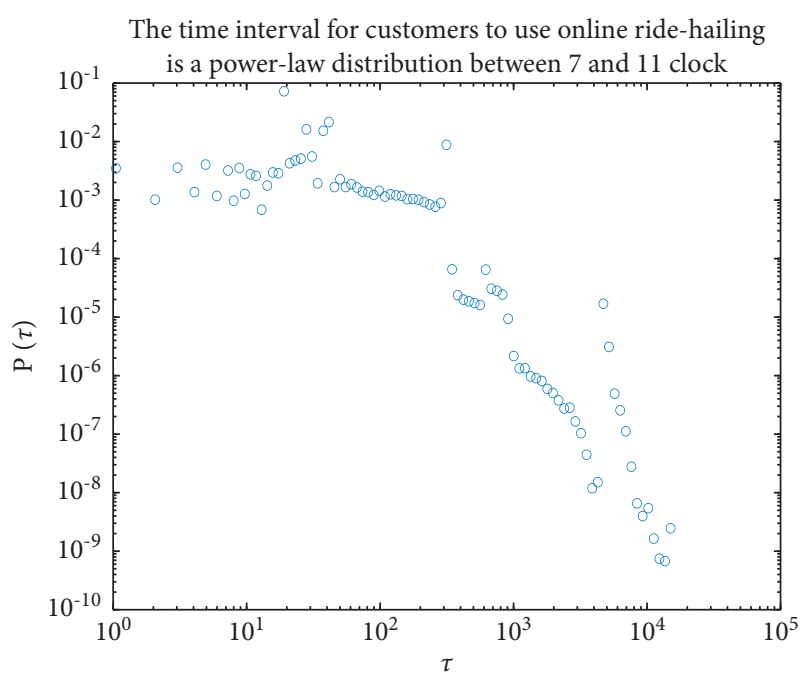

(a)



(b)

FIgURE 7: Two-segment power-law distribution of e-hailing vehicles.

area number, positioning time, longitude, latitude, speed, and travel number. The detailed description of the specific fields is shown in Table 2.

Before the track data are mined and analyzed, the original data should be classified, summarized, cleaned, and sorted according to the data structure requirements of the input part according to the method proposed in this paper. The redundant data and noise data should be removed. When the vehicle is in a static state, a large number of redundant points will be generated. For example, repeated track points will be generated when the car is not started or when the vehicle stops at a particular place during a specific period on the road. As the method proposed in this paper requires path discovery within each selected time interval, the time interval is set as one hour according to the available usage time of online hailing vehicles. The specific data processing process is as follows: Use the Pandas library to sort the track data by license plate number and location time, or sort by order number and location time, and sort the movement trajectory of each online booking car. Further, the redundant points in the track data of each online hailing car are removed (or the data with the order number 0 ). Since the data types of OsitionTime and UpdateTime are timestamping, which is not conducive to relevant time calculation, we convert the timestamp format to data time type.

4.4. Critical Analysis of Activity Grouping. Activity refers to the frequency with which particular behaviour occurs in a certain period. The higher the frequency is, the more active the user will be; the lower the frequency is, the less active the user will be. 



FIGURE 8: The time interval distribution of ride-hailing services of different companies in the use of two adjacent times.

TABLE 2: Online ride-hailing trajectory data set of a northern city in China.

\begin{tabular}{|c|c|c|}
\hline Field names & Field type & Field description \\
\hline Guide & Int & Track ID \\
\hline Company & Varchar & The company ID \\
\hline Vehicle & Varchar & License plate number \\
\hline VehicleRegioncode & Int & Vehicle area number \\
\hline PositionTime & Varchar & Location timestamp \\
\hline UpdateTime & Varchar & Locates the update timestamp \\
\hline Long & Float & $\begin{array}{l}\text { Longitude at the time of } \\
\text { positioning }\end{array}$ \\
\hline Latitude & Float & Latitude at the time positioning \\
\hline Speed & Int & Vehicle speed \\
\hline Ordered & Int & Trip ID \\
\hline
\end{tabular}

In previous empirical studies, individuals with different activity levels will regularly change their corresponding statistical characteristics. In this section, users are grouped according to the number of times they have used shared bikes. Table 3 shows the activity statistics after the subtables.

In Table 3, the maximum number of users using shared bikes during this period is 34 , and the minimum number is only one. The total number of users is 972,521 . As can be seen from Table 3, the number of users who use for 1-3 times is the most, and the number of users who use for 19-27 times is the least. The fewer the number of users, the more the uses.

In Figure 9, triangles, circles, squares, rhombus, and inverted triangles are grouped from more minor to more. As shown in Figure 9, the more frequent the use, the more pronounced the bimodal phenomenon in the time interval of shared bikes. With the increase of the number of users, the whole function curve goes up, and the extreme value of the two peaks is the highest. The less frequently used group at the end of the second half, the higher the curve.

Here, we found a phenomenon different from previous empirical studies on activity conducted by other scholars. They found that there was a positive correlation between people's movement and power index $\alpha$. We take the function curve $\tau>730$, the second half of $\tau>730$ curve was fitted, and its power index was calculated. We can see this in Figure 9 that, $\tau>$ the latter part of the curve of 730 does not show a simple positive correlation, and the last part of the function is truncated at about $\tau=84300$. After $\tau \geq 84300$ (i.e., close to 23.4 hours), the power index of $730 \leq \tau \leq 84300$ keeps a positive correlation while instantly increases significantly.

4.5. Analysis of Episodic Memory. Whether seen from human communication modes such as e-mail or natural phenomena such as earthquakes, their behaviours often 
TABLE 3: Activity grouping table.

\begin{tabular}{lccccc}
\hline Category & Group 1 & Group 2 & Group 3 & Group 4 & Group 5 \\
\hline User number & 843999 & 86115 & 25292 & 16040 & 1073 \\
Number of uses & $1-3$ times & $4-6$ times & $7-9$ times & $10-18$ times & $19-27$ times \\
\hline
\end{tabular}

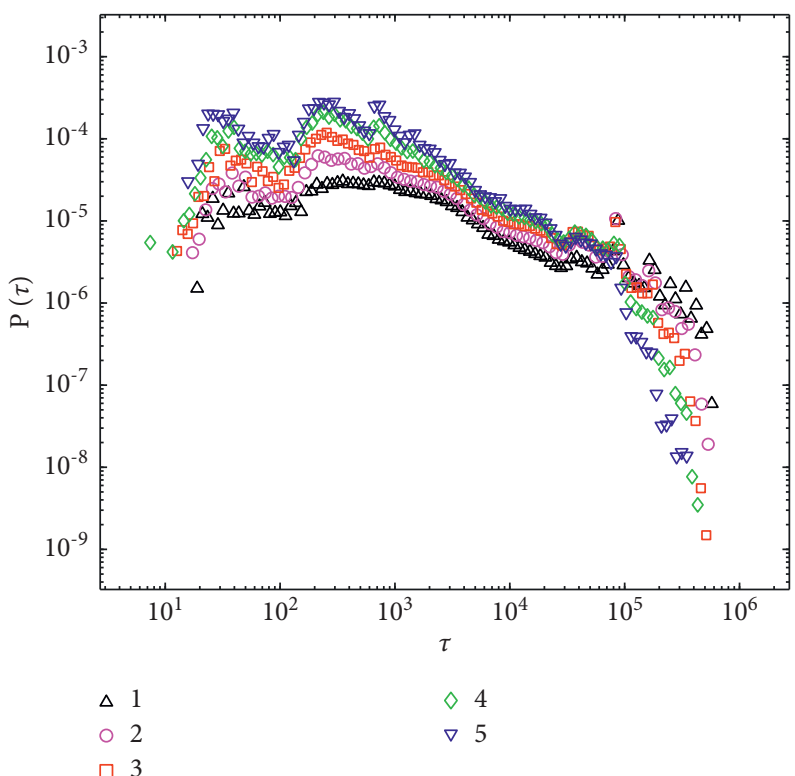

Figure 9: The relationship between the number of users using online ride-hailing and the power index.

show short-term concentrated bursts and long periods of silence, which we call the paraphernalia of human behaviours [37]. Most of the time intervals are smaller than the average time interval, and some considerable time intervals also occur, resulting in a significant standard deviation of the time interval distribution. To study the outbreak of Posting behaviour in QZONE, we will group users according to their monthly average posting volume and make corresponding BM phase diagrams. First, we define the analytic index of temporal paroxysm. Literature [38] proposed an index to describe the frenzy of interval time:

$$
B=\frac{\left(\sigma_{\tau} / v\right)}{\left(\sigma_{\tau} / m_{\tau}+1\right)}=\frac{\left(\sigma_{\tau}-m_{\tau}\right)}{\left(\sigma_{\tau}+m_{\tau}\right)}
$$

where $\sigma_{\tau}$ represents the standard deviation of the interval time, $m_{\tau}$ represents the expectation of the interval time, which is easily obtained from (1) $-1 \leq B \leq 1, B=1$ means that the event has proxy-like solid behaviour, $B=0$ means that an event is a random event obeying Poisson behaviour, and $B=-1$ implies that the time of the periodic event is regular.

For example, in Nature, the time and place of extreme climate have a strong memory. When we order the time interval of events, if a long time interval is followed by a long time interval with a considerable probability, and vice versa. When a short time interval is followed by a short time interval with a significant likelihood, this type of schedule shows good memorability; otherwise, it shows weak or antimemorability. This sequence consists of $n_{\tau}$ elements (meaning that $n_{\tau}-1$ behaviour occurs), and the first $n_{\tau}-1$ element is specified to form sequence 1 and the last $n_{\tau}-1$ element is specified to form sequence 2. As shown in equation (3), the Pearson correlation between these two sequences can be used to measure the memory of this sequence [38].

Among them, the memory coefficient of interval time is as follows [27]:

$$
M=\frac{1}{n_{\tau}-1} \sum_{i=1}^{n_{\tau}-1} \frac{\left(\tau_{i}-m_{1}\right)\left(\tau_{i+1}-m_{2}\right)}{\left(\sigma_{1} \sigma_{2}\right)}
$$

where $M_{1}$ and $m_{2}$ are the mean values of sequence one and sequence two and $\sigma_{1}$ and $\sigma_{2}$ are the standard deviations of sequence 1 and sequence 2 , respectively. $M$ as a biased estimate for the autocorrelation function; its scope is in $(1,1)$. When $M$ is close to 1 , the interval time of the same behavior is usually long interval time and long interval time, and short interval time and short interval time appear successively. Neutral close to 0 , when $M$ is close to -1, the interval of the same behavior is usually long interval and short interval, and the short interval and long interval appear successively.

We mainly analyze the temporal behaviour patterns of users using the ride-hailing services of different ride-hailing companies in terms of episodic memory. The following table shows the number of users who have used the online ridehailing service of other companies from 1 to 24 times, and the details are shown in Table 4.

The data set we used here actually owns 12 ride-hailing companies, among which the data volume of eight companies is too small, which makes the data analysis less authentic. Therefore, we did not analyze this experiment. In this experiment, we use four data quantities of the biggest companies, respectively, as the company $\mathrm{A}$, company $\mathrm{B}$, company $\mathrm{C}$, and 
TABLE 4: The number of people from different companies who have obtained online rides from 1 to 24 times.

\begin{tabular}{|c|c|c|c|c|}
\hline Number of uses & Company A & Company B & Company C & Company D \\
\hline 1 & 576 & 1225948 & 3429 & 2863 \\
\hline 2 & 277363 & 24820 & 13122 & 3524586 \\
\hline 3 & 0 & 8191 & 1268 & 6 \\
\hline 4 & 927 & 3865 & 2319 & 10925 \\
\hline 5 & 0 & 852 & 1034 & 0 \\
\hline 6 & 114 & 262 & 658 & 53 \\
\hline 7 & 0 & 225 & 678 & 0 \\
\hline 8 & 85 & 2 & 3898 & 4 \\
\hline 9 & 0 & 204 & 2025 & 0 \\
\hline 10 & 79 & 2918 & 12 & 0 \\
\hline 11 & 0 & 20 & 0 & 0 \\
\hline 12 & 30 & 10 & 0 & 0 \\
\hline 13 & 0 & 3 & 0 & 0 \\
\hline 14 & 26 & 2 & 2 & 1 \\
\hline 15 & 0 & 0 & 0 & 0 \\
\hline 16 & 14 & 0 & 0 & 0 \\
\hline 17 & 0 & 0 & 0 & 0 \\
\hline 18 & 9 & 0 & 2 & 0 \\
\hline 19 & 0 & 1 & 0 & 0 \\
\hline 20 & 7 & 0 & 1 & 0 \\
\hline 21 & 0 & 0 & 0 & 0 \\
\hline 22 & 1 & 0 & 0 & 0 \\
\hline 23 & 0 & 0 & 0 & 0 \\
\hline
\end{tabular}

company $\mathrm{D}$. Then, we analyze the temporal regularity and memory of users' behaviour patterns using the online hailing vehicles of four companies and find that the users have different episodic memories when using other online hailing cars. The following four graphs, respectively, correspond to the BM chart of paroxymoron and memory of users using the online ride-hailing service of companies $\mathrm{A}, \mathrm{B}, \mathrm{C}$, and $\mathrm{D}$.

Figure 10(a) BM plot presents an inverted "J" shape. The head and tail points of "J" are dense, while the middle connecting part points are relatively sparse. The density points of the head and tail are about $M=-0.11, B=0.39$ : $M=0.38$, and $B=-0.58$, respectively. Figure $10(\mathrm{~b})$ shows a central phenomenon near $M=-0.17$ and $b=-0.35$. Because the company's data volume is small, few points are in Figure $10(\mathrm{c})$. Although there are few points, it is easier for us to find the values of $\mathrm{M}$ and $\mathrm{B}$. Therefore, we can think of these few points as a point that the users of company ridehailing are a specific group of people, and their episodic and memorable behaviour characteristics are almost the same. The memory coefficient of the formation method in Figure $10(\mathrm{c})$ is $M=0.002$ and $B=0.856$. The points in Figure $10(d)$ are relatively scattered, and the range of the matrix memory coefficient is mainly between $-0.8 \leq M \leq 0.78$ and $-0.77 \leq B \leq 0.51$.

In previous time characteristic of human behaviour motivation on the study of the law of memory, most present a centralized (pictured above Figures 10(b) and 10(c)) or law offers a planar scattered memory (shown above Figure 10(d)) and form two centralized, two groups of BM coefficient value of the very few, so it is quite possible that the experimental phenomenon shown in Figure 10 is a completely new discovery. It is revealed that the formation memory characteristics under the same behaviour mode are not necessarily unique, and there is only one set of BM coefficient values.
As for the time behaviour pattern of users using shared bikes, the episodic memory is briefly discussed here. The points in Figure 11 are also similar to Figure 10(d), and the distribution is relatively divergent. The range of the matrix memory coefficient is mainly $-0.791 \leq M \leq 0.747$ and $-0.547 \leq B \leq 0.405$.

4.6. Integration Analysis of the Urban Spatial Structure and Human Traffic Behaviour Pattern. It has become an essential topic in the research of computational sociology to use electronic footprint big data to conduct quantitative analysis of human movement behaviour to explore human behaviour patterns and urban operation law. In this part, we take the city as an example and analyze the relationship and connection with the urban spatial structure from the spatial motion trajectory using the track data of online ride-hailing.

As can be seen from Figure 12, the car-hailing tracks are distributed mainly around the radiation network of some administrative areas of different levels. The higher the organizational level, the denser the car-hailing ways and the larger the radiation surface. On the contrary, the lower the administrative level, the higher the coefficient of the ride-hailing track and the smaller the radiation surface. The general trajectory of online ride-hailing shows a radiating network distribution. The so-called "network" is based on a traffic line (such as ordinary provincial, county, township roads, and expressways) and around an essential urban regional structure (residential, industrial, commercial, administrative, cultural, tourist, and green areas, etc.). The company's ride-hailing track data follows a highway around a prefecture-level city in the track chart above. 


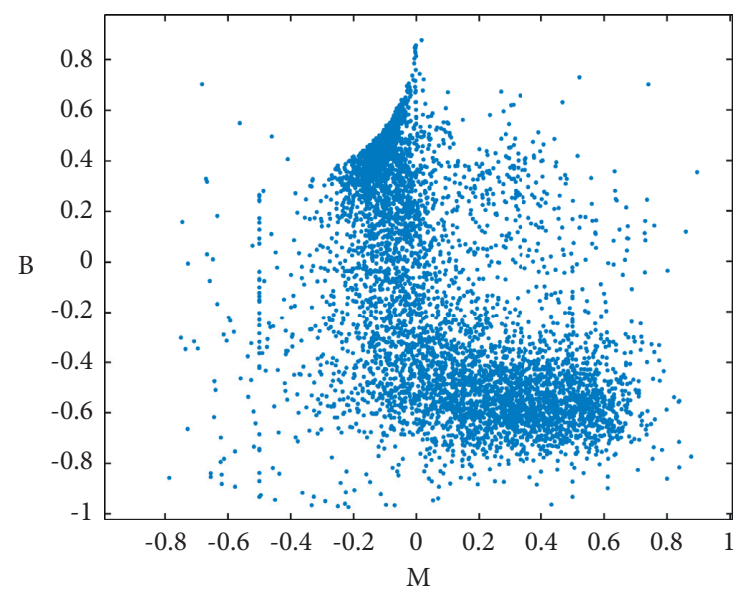

(a)



(c)

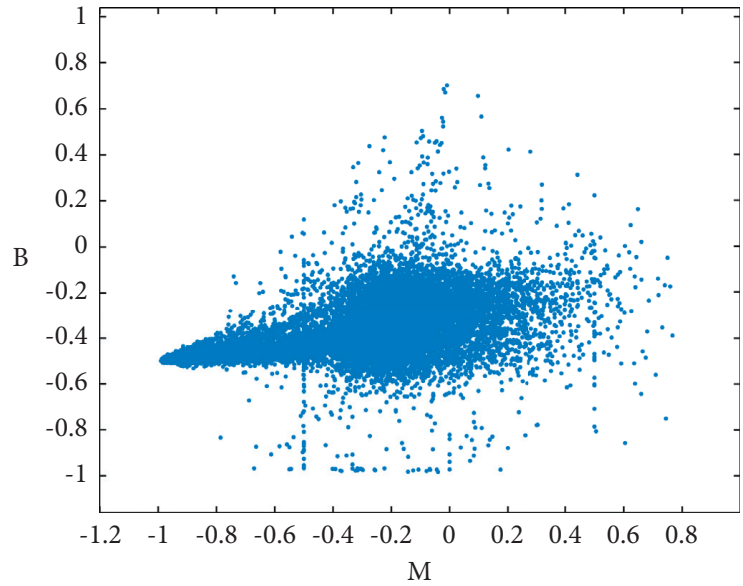

(b)

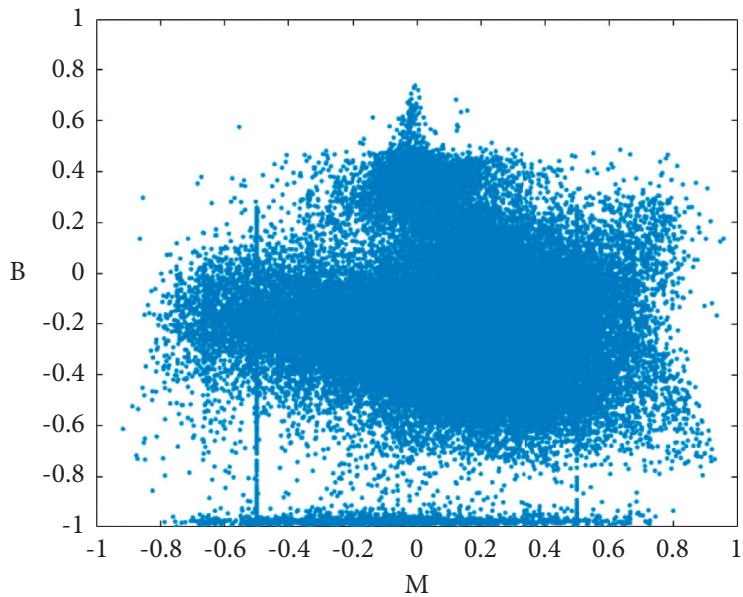

(d)

Figure 10: BM chart of the four companies with the most comprehensive data volume. (a) Company A. (b) Company B. (c) Company C. (d) Company D.



FIgURE 11: BM chart of bike-sharing users. 


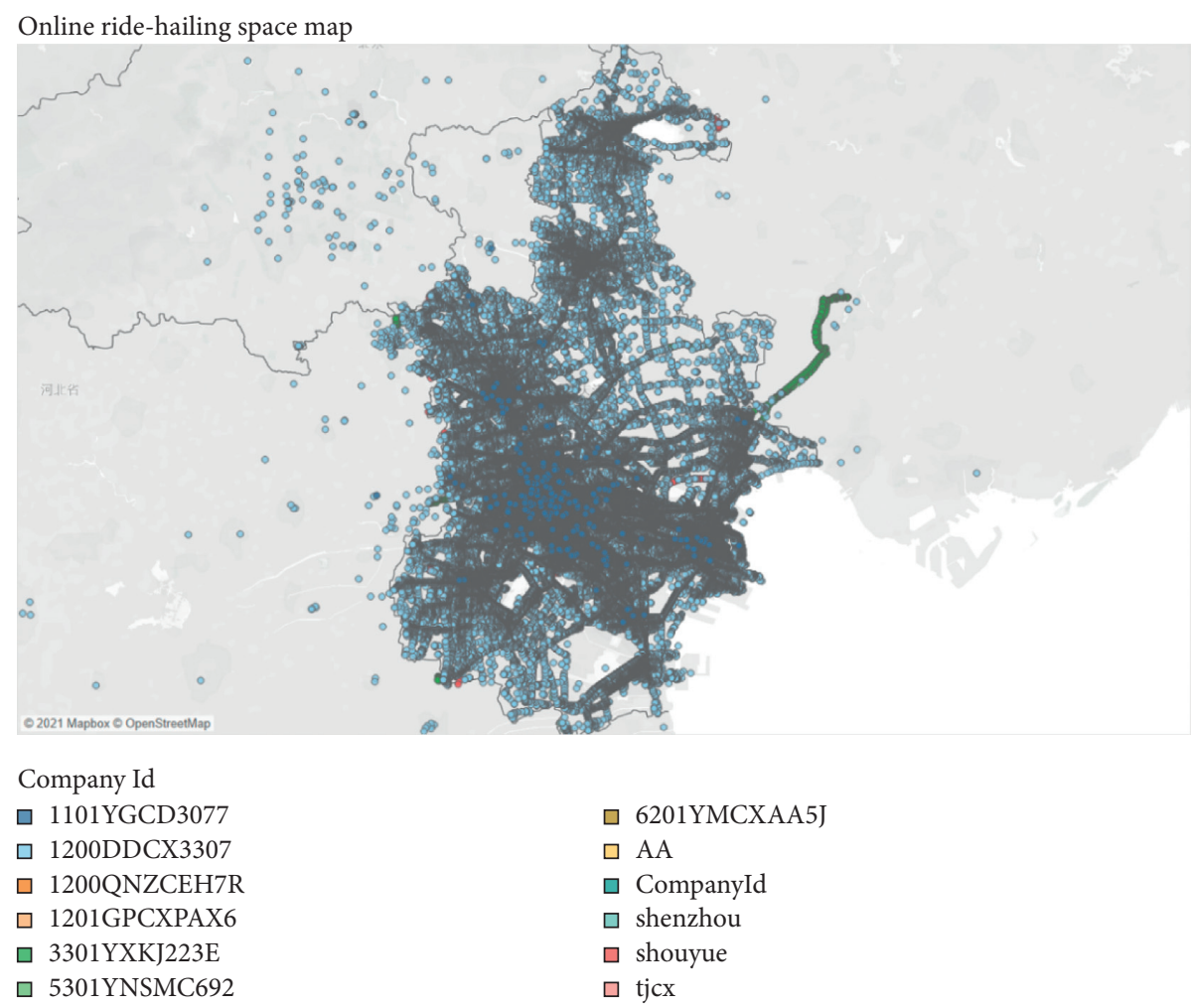

Figure 12: Net about car path chart.

\section{Conclusions}

With the acceleration of urbanization, the number of motor vehicles and the frequency of use have risen sharply, and the urban traffic congestion problem has become more and more prominent. In the past, it has been a challenge to ease the supply of transport facilities simply by building transport facilities and expanding new roads to alleviate demand. This paper uses bike-sharing and ride-hailing data from a city in China to examine cycle and volatility and time interval. Traffic behaviour dynamics under multiple traffic modes, such as urban spatial structure and human traffic behaviour patterns, are studied. The experimental results show that users using shared cycling and web about car distribution in the interval between two double logarithmic coordinate systems present a power-law distribution. The user uses shared cycling on the time interval between two times of power-law distribution shows relatively prominent bimodal distribution characteristics. On the web, the car data set does not have this kind of phenomenon. A typical power-law distribution is presented in the time interval between two adjacent ride-hailing services. It is found that the power index of users is different when they choose additional ride-hailing services. There is a positive correlation between the user's activity and the powerlaw bimodal phenomenon in the double logarithmic coordinate system. The stronger the action is, the higher the peak shape and the extreme value of the two peaks are and the peak shape is different under different activities. The lower the action is, the less noticeable the bimodal phenomenon is. Finally, we analyze the characteristics of the city's urban spatial structure and find a close relationship between the behaviour pattern of people, whether using shared bikes or online ride-hailing, and the urban spatial design. The experimental results can provide planning support for constructing a smart city by analyzing the behaviour model.

\section{Data Availability}

The data mainly refer to the bike-sharing and ride-hailing data of a developed city in China, which cannot be disclosed to the public at the partner's request.

\section{Conflicts of Interest}

The authors declare that they have no conflicts of interest or personal relationships that could have appeared to influence the work reported in this paper.

\section{Authors' Contributions}

Hongwei Jing was mainly responsible for data collating and writing. Xiaoming Li mainly modified and polished the article. Guangquan Xu designed the paper framework and the model. Mengli Zhu and Haoyang Peng primarily designed the thesis program. Li Shen and FangYuan Liu got data and the results verified.

\section{Acknowledgments}

This work was partially sponsored by the National Key R\&D Program of China (no. 2019YFB2101700), the National Science Foundation of China (62172297 and 61902276), the 
Key Research and Development Project of Sichuan Province (no. 21SYSX0082), the Tianjin Intelligent Manufacturing Special Fund Project (20201159), and the Key Research and 635 Development Project of Xinjiang Production and Construction Corps 12th (no. SR202103).

\section{References}

[1] A.-L. Barabási, "The origin of bursts and heavy tails in human dynamics," Nature, vol. 435, no. 7039, pp. 207-211, 2005.

[2] D. Brockmann, L. Hufnagel, and T. Geisel, "The scaling laws of human travel," Nature, vol. 439, no. 7075, pp. 462-465, 2006.

[3] X. P. Han, B. H. Wang, and T. Zhou, "Researches of human dynamics," Complex System and Complexity Science, vol. 7, no. 2, pp. 132-144, 2010, in Chinese.

[4] D. Lazer, A. Pentland, L. Adamic et al., "computational social science," Science, vol. 323, no. 5915, pp. 721-723, 2009.

[5] P. Blanchard and M. O. Hongler, "Modeling human activity in the spirit of Barabasi's queueing systems," Physical review. E, Statistical, nonlinear, and soft matter physics, vol. 75, no. 2, Article ID 026102, 2007.

[6] G. Xu, Y. Zhao, L. Jiao et al., "An efficient sparse decision making model with two-way trust recommendation in the AI enabled IoT systems," IEEE Internet of Things Journal (Early Access), p. 1, 2020.

[7] J. G. Oliveira and A. Vazquez, "Impact of interactions on human dynamics," Physica A: Statistical Mechanics and Its Applications, vol. 388, no. 2-3, pp. 187-192, 2009.

[8] X. Li, G. Xu, C. Liu et al., "High order feature modelling of dynamic network nodes based on social network security," Future Generation Computer Systems, vol. 125, no. 8, 2021.

[9] A. Vazquez, "Impact of memory on human dynamics," Physica A: Statistical Mechanics and Its Applications, vol. 373, pp. 747-752, 2007.

[10] R. D. Malmgren, D. B. Stouffer, A. E. Motter, and L. A. N. Amaral, "A Poissonian explanation for heavy tails in e-mail communication," Proceedings of the National Academy of Sciences, vol. 105, no. 47, pp. 18153-18158, 2008.

[11] R. D. Malmgren, D. B. Stouffer, A. S. L. O. Campanharo, and L. A. N. Amaral, "On universality in human correspondence activity,” Science, vol. 325, no. 5948, pp. 1696-1700, 2009.

[12] S. Quinn, N. Murphy, and A. F. Smeaton, "Tracking human behavioural consistency by analyzing periodicity of household water consumption," in Proceedings of the 2019 2nd International Conference on Sensors, Signal and Image Processing, pp. 1-5, Prague Czech Republic, October 2019.

[13] T. Zhou, H. A. Kiet, B. J. Kim, B. H. Wang, and P. Holme, "Role of activity in human dynamics," Europhysics Letters, vol. 82, no. 2, p. 28002, 2008.

[14] W. Hong, X. P. Han, T. Zhou, and B. H. Wang, "Heavy-tailed statistics in short-message communication," Chinese Physics Letters, vol. 262, no. 2, Article ID 028902, 2009.

[15] P. Wang, X.-Y. Xie, C. H. Yeung, and B.-H. Wang, "Heterogenous scaling in the inter-event time of on-line bookmarking," Physica A: Statistical Mechanics and Its Applications, vol. 390, no. 12, pp. 2395-2400, 2011.

[16] Z. Huang, P. Wang, F. Zhang, J. Gao, and M. Schich, "A mobility network approach to identify and anticipate large crowd gatherings," Transportation Research Part B: Methodological, vol. 114, pp. 147-170, 2018.

[17] P. Thakuriah, "Urban transportation planning: a decisionoriented approach," Journal of Transportation Engineering, vol. 127, no. 5, p. 454, 2001.
[18] F. Giannotti, M. Nanni, D. Pedreschi et al., "Unveiling the complexity of human mobility by querying and mining massive trajectory data," The VLDB Journal, vol. 20, no. 5, pp. 695-719, 2011.

[19] I. Rhee, M. Shin, S. Hong, K. Lee, S. J. Kim, and S. Chong, "On the levy-walk nature of human mobility," IEEE/ACM Transactions on Networking, vol. 19, no. 3, pp. 630-643, 2011.

[20] C. Li and Z. Wang, "Method on layout optimizing of public bicycle rental stations based on complex network theory," in Proceedings of the Third International Conference on Transportation Engineering (ICTE), pp. 247-252, Chengdu, China, July 2011.

[21] Y. Peng, T. Liang, Y. Yang, H. Lin, P. Li, and J. Deng, "A key node optimization scheme for public bicycles based on wavefront theory," The International Journal on Artificial Intelligence Tools, vol. 29, p. 07n08, Article ID 2040016, 2020.

[22] Z. Gao, S. Wei, L. Wang, and S. Fan, "Exploring the spatialtemporal characteristics of traditional public bicycle use in Yancheng, China: a perspective of time series cluster of stations," Sustainability, vol. 12, no. 16, p. 6370, 2020.

[23] W. Zi, W. Xiong, H. Chen, and L. Chen, "TAGCN: stationlevel demand prediction for bike-sharing system via a temporal attention graph convolution network," Information Sciences, vol. 561, pp. 274-285, 2021.

[24] B. Chen, S. Zhou, H. Liu et al., "A prediction model of online car-hailing demand based on K-means and SVR," Journal of Physics: Conference Series, vol. 1670, no. 1, Article ID 012034, 2020.

[25] X. W. Jia, "Characteristics analysis and demand prediction of travel demand based on online car-hailing data," Journal of Transportation Engineering, vol. 18, no. 5, pp. 39-45, 2018.

[26] S. Jiang, W. Chen, Z. Li, and H. Yu, "Short-term demand prediction method for online car-hailing services based on a least squares support vector machine," IEEE Access, vol. 7, pp. 11882-11891, 2019.

[27] M. H. C. Barboza, R. d. S. Alencar, J. C. Chaves, M. A. H. B. Silva, R. D. Orrico, and A. G. Evsukoff, "Identifying human mobility patterns in the Rio de Janeiro metropolitan area using call detail records," Transportation Research Record: Journal of the Transportation Research Board, vol. 2675, no. 4, pp. 213-221, 2021.

[28] C. Zhao, A. Zeng, and C. H. Yeung, "Characteristics of human mobility patterns revealed by high-frequency cell-phone position data," EPJ Data Science, vol. 10, no. 1, p. 5, 2021.

[29] S. Hu, S. Gao, L. Wu et al., "Urban function classification at road segment level using taxi trajectory data: a graph convolutional neural network approach," Computers, Environment and Urban Systems, vol. 87, Article ID 101619, 2021.

[30] S. Siangsuebchart, S. Ninsawat, A. Witayangkurn, and S. Pravinvongvuth, "Public transport GPS probe and rail gate data for assessing the pattern of human mobility in the Bangkok Metropolitan Region, Thailand," Sustainability, vol. 13, no. 4, p. $2178,2021$.

[31] K. Liu, Y. Murayama, and T. Ichinose, "Exploring the relationship between functional urban polycentricity and the regional characteristics of human mobility: a multi-view analysis in the Tokyo metropolitan area," Cities, vol. 111, Article ID 103109, 2021.

[32] J. Li, T. Cai, K. Deng, X. Wang, T. Sellis, and F. Xia, "Community-diversified influence maximization in social networks," Information Systems, vol. 92, Article ID 101522, 2020.

[33] A. Noulas, S. Scellato, R. Lambiotte, M. Pontil, and C. Mascolo, "A tale of many cities: universal patterns in 
human urban mobility," PLoS ONE, vol. 7, no. 5, Article ID e37027, 2012.

[34] Y. Huang, Z. Xiao, X. Yu, D. Wang, V. Havyarimana, and J. Bai, "Road network construction with complex intersections based on sparsely sampled private car trajectory data," ACM Transactions on Knowledge Discovery from Data, vol. 13, no. 3, pp. 1-28, 2019.

[35] S. Milojević, "Power law distributions in information science: making the case for logarithmic binning," Journal of the American Society for Information Science and Technology, vol. 61, no. 12, pp. 2417-2425, 2010.

[36] R. Harang and A. Kott, "Burstiness of intrusion detection process: empirical evidence and a modeling approach," IEEE Transactions on Information Forensics and Security, vol. 12, no. 10, pp. 2348-2359, 2017.

[37] K. I. Goh and A. L. Barabási, "Burstiness and memory in complex systems," Europhysics Letters, vol. 81, no. 4, p. 48002, 2008.

[38] X. P. Han, T. Zhou, and B. H. Wang, "Modeling human dynamics with adaptive interest," New Journal of Physics, vol. 10, Article ID 073010, 2008. 